\title{
ANALYTIC ROOT LOCUS AND LAMBERT $W$ FUNCTION IN CONTROL OF A PROCESS WITH TIME DELAY
}

\author{
Brian COGAN * Annraoi M de PAOR ${ }^{* *}$
}

\begin{abstract}
Recently, the Lambert $W$ function has arisen in the analysis of many systems including a restricted class of time-delay systems. An alternative approach to this analysis, based on the well-established root locus method, is shown here to contain the Lambert $\mathrm{W}$ function as a special case.

As a purely illustrative example of the equivalence between the Lambert $W$ function and analytic root locus a system comprising a Proportional controller with a time-delay process is analysed. Controller designs based on rightmost eigenvalue location and the dominant eigenvalue method are described.
\end{abstract}

K e y w or d s: Lambert $W$ function, root locus, time-delay system, stability, eigenvalues

\section{ORIGIN OF THE LAMBERT $W$ FUNCTION}

Johann Heinrich Lambert (1728-1777) was a multitalented scientist and philosopher who produced important work in fields such as number theory, optics, meteorology, and astronomy - to name but a few. Some notes on Lambert's life and work may be found in [1]. For further biographical information and details of Lambert's contributions to the mathematical theory of perspective see $[2]$.

In 1758 Lambert derived a series solution to the trinomial equation

$$
x=q+x^{m} .
$$

In 1779 Euler studied the following transformed version of (1)

$$
x^{\alpha}-x^{\beta}=(\alpha-\beta) v x^{\alpha+\beta} .
$$

When considering a special case of his series solution to (2) Euler introduced a function $w$ that satisfies

$$
w e^{-w}=x .
$$

A related function $W(z)$ that solves

$$
W(z) e^{W(z)}=z
$$

has become know as the Lambert $W$ function. References to original papers and further details of these derivations may be found in [1].

The Lambert $W$ function has found applications in an astonishing diversity of disciplines including celestial mechanics [3], electromagnetism [4], supply chain dynamics [5], quantum theory [6, 7], and solar physics [8]. Typically, the Lambert $W$ function is used in these references to derive closed-form solutions where previously this was not possible. Computer algebra systems such as Maple [9], Matlab [10], and Mathematica [11] implement the Lambert $W$ function.

\section{ANALYTIC METHOD FOR DRAWING ROOT LOCUS PLOTS FOR SYSTEMS WITH TIME DELAY}

If the process $G(s)$ has time delay $L>0$ the characteristic polynomial of the system in Fig. 1 is

$$
p(s)=N(s)+k e^{-s L} M(s)
$$

where $N(s)$ and $M(s)$ are polynomials of degree $n$ and $m$ respectively, with $m \leq n, s=\sigma+j \omega$ and the gain $k$ is a real number. The roots of (5) are the eigenvalues of the system. In root locus terminology, the poles and zeros of the root locus are given by the roots of $N(s)=0$ and $M(s)=0$, respectively. The root locus of (5) consists of the paths traced out in the $(\sigma, \omega)$ plane by the roots of (5) as $k$ varies and these paths are called the root locus branches. As for the delay-free case, the root loci for time-delay systems are symmetrical about the real axis, but unlike the delay-free case, the number of root locus branches is infinite. Setting $k=0$, these branches start at the poles (roots of $N(s)=0$ ) and at $\sigma=-\infty$; setting $k= \pm \infty$, these branches terminate at the zeros (roots of $M(s)=0)$ and at $\sigma=+\infty$. Root locus branches that do not terminate at a zero approach $\sigma=+\infty$ along asymptotes. These asymptotes are infinite in number and parallel to the real axis.

A geometric method, consisting of rules for drawing the root locus of (5) when $L=0$ may be found in standard texts such as [12]. An extended set of these rules for drawing the root locus of (5) with $L>0$ may be found in [13-15]. Alternatively, when $L=0$ there is an analytic method $[16,17]$ for drawing the root locus of (5). This method consists of deriving an equation for the root locus curves. A root locus equation for $L>0$ will now be derived. In the following the real and imaginary parts of the polynomial $P$ are written as $\operatorname{Re} P$ and $\operatorname{Im} P$ respectively and $e^{-s L} M(s)$ is written as $\hat{M}(s)$.

\footnotetext{
* Commissioners of Irish Lights, Harbour Road, Dun Laoghaire, Co. Dublin, Ireland, b.cogan@cil.ie; ${ }^{* *}$ School of Electrical, Electronic and Mechanical Engineering, National University of Ireland, Dublin, Belfield, Dublin 4, Ireland, annraoi.depaor@ucd.ie
} 


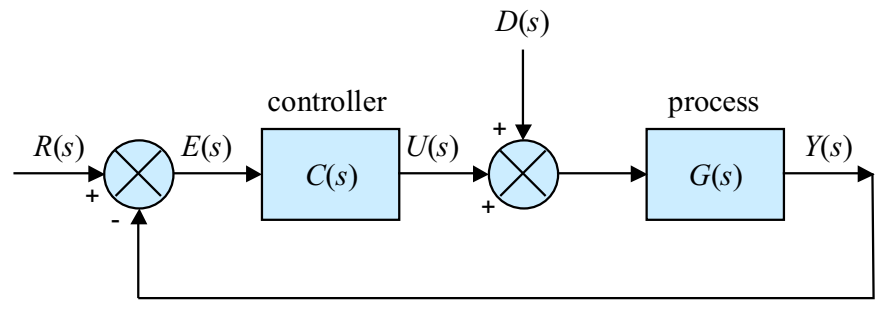

Fig. 1. Fig. 1 Standard unity negative feedback system. $D(s)$ represents disturbance signals and $R(s)$ represents the reference signal.

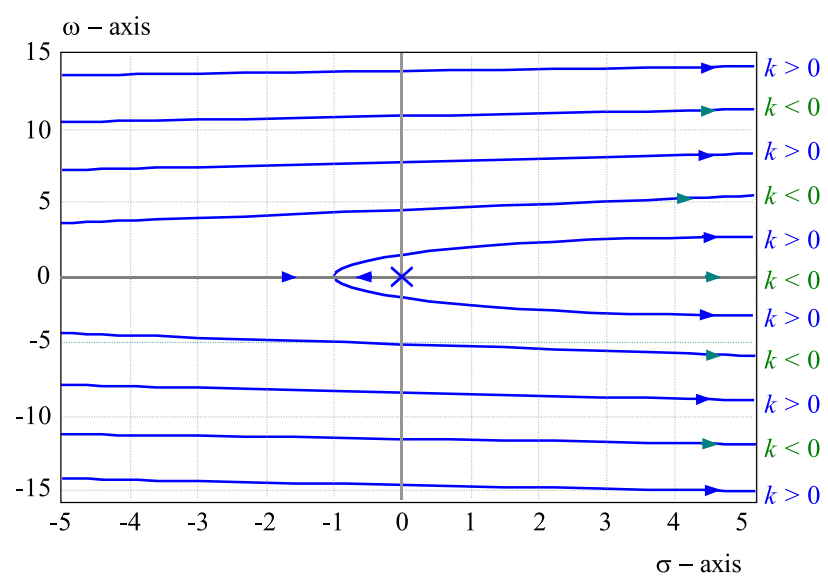

Fig. 2. Illustrative example of a root locus diagram for a time-delay system.

Equation (5) may be rewritten as

$$
p(s)=N(s)+k \hat{M}(s)=0 .
$$

(6) can be rearranged as $k=-\frac{N(s)}{\hat{M}(s)}=\frac{-\operatorname{Re} N-j \operatorname{Im} N}{\operatorname{Re} \hat{M}+j \operatorname{Im} \hat{M}}$ or

$k=\frac{-\operatorname{Re} N \operatorname{Re} \hat{M}-\operatorname{Im} N \operatorname{Im} \hat{M}+j(\operatorname{Re} N \operatorname{Im} \hat{M}-\operatorname{Im} N \operatorname{Re} \hat{M})}{\left.(\operatorname{Re} \hat{M})^{2}+\operatorname{Im} \hat{N}\right)^{2}}$

If $k$ is a real number (7) gives the root locus equation

$$
\operatorname{Re} N \operatorname{Im} \hat{M}-\operatorname{Im} N \operatorname{Re} \hat{M}=0 .
$$

The plot of $(8)$ in the $(\sigma, \omega)$ plane gives the entire root locus of (5) for $-\infty<k<\infty$, that is, (8) contains both the positive gain root locus, corresponding to $k>0$, as well as the negative gain root locus, corresponding to $k<0$. Given the root locus equation (8), the gain equation (7) becomes

$$
k=\frac{-\operatorname{Re} N \operatorname{Re} \hat{M}-\operatorname{Im} N \operatorname{Im} \hat{M}}{(\operatorname{Re} \hat{M})^{2}+(\operatorname{Im} \hat{M})^{2}} .
$$

(9) describes a set of curves in the $(\sigma, \omega)$-plane. For a given value of $k$ these curves intersect the root locus curves at the eigenvalues of (6) corresponding to that value of $k$.

For a different and less general analytic approach to root locus of certain time-delay systems see [18].
EXAMPLE 1. As an example of the use of (8) and (9) consider the characteristic equation of a system comprising a proportional controller and an integrator with unit time delay [15]

$$
s+k e^{-s}=0 .
$$

Using (8) we can say that (10) has root locus equation

$$
\omega \cos \omega+\sigma \sin \omega=0
$$

Using (9) we can say that (10) has gain equation

$$
e^{\sigma}(\omega \sin \omega-\sigma \cos \omega)=k .
$$

Equation (11), the root locus equation for (10), is plotted in Fig. 2. Other examples of root locus diagrams for timedelay systems may be found in [18-20].

Figure 2 shows the root locus diagram for a system with characteristic equation $p(s)=s+k e^{-s}, k \in \Re^{1}$, $i e$ proportional control of a single integrator with unity time delay. The lines are the paths of the eigenvalues as $k$ varies and these paths are referred to as the root locus branches. Arrows indicate the direction of the eigenvalues with increasing $|k|$. There is a pole at $s=0$ (indicated by an " $\times ")$ - a root locus branch starts at this pole when $k=0$. This branch meets another branch coming from $\sigma=-\infty$ to form a breakpoint. These two branches then leave the $\sigma$-axis, cross the imaginary axis and approach $\sigma=\infty$. In addition there is an infinite number of other root locus branches that are parallel to the real axis and start at $\sigma=-\infty$ and approach $\sigma=\infty$ along asymptotes. If, for a chosen value of $k$, a plot of the gain equation (12) were superimposed onto Fig. 2 we would see another curve intersecting the root locus branches orthogonally at the eigenvalues associated with the chosen value of $k$.

EXAmPLE 2. For another example of a root locus diagram consider Fig. 3 [19,20].

Figure 3 shows the primary branches of the root locus of $p(s)=s(s+1 / \tau)+k(s+F) e^{-s}-$ the characteristic equation for a PI controller $C(s)=k_{2}(1+F / s)$ with a first-order lag plus time-delay (FOLPD) process: $G(s)=$ $k_{1} e^{-s L} /(s+1 / \tau)$. In Fig. 3 we have used $k_{1} k_{2}=k>$ $0, L=1, \tau=5$ and $F=0.25$. As is conventional, poles are indicated by " $\times$ "'s and the zero is indicated by a " $\bigcirc$ ". A branch starts at each of the poles $s=$ 0 and $s=-1 / \tau$. One of these branches ends at the zero at $s=-F$. The other branch travels to the left 
Table 1. Root locus equations and gain equations derived using equations (8) and (9) respectively.

\begin{tabular}{lll}
\hline polynomial & Root locus equation & Gain equation \\
\hline$s+k e^{-s}$ & $\omega \cos \omega+\sigma \sin \omega=0$ & $e^{\sigma}(-\sigma \cos \omega+\omega \sin \omega)$ \\
$(s+a)+k e^{-s}$ & $\omega \cos \omega+(a+\sigma) \sin \omega=0$ & $e^{\sigma}(-(a+\sigma) \cos \omega+\omega \sin \omega)$ \\
$(s+a)+k s^{2} e^{-s}$ & $\omega\left(2 a \sigma+\sigma^{2}+\omega^{2}\right) \cos \omega$ & $\frac{-e^{\sigma}}{\left(\sigma^{2}+\omega^{2}\right)^{2}}\left[\left(\sigma^{2}(a+\sigma)+(\sigma-a) \omega^{2}\right) \cos \omega\right.$ \\
& $-\left(a \sigma^{2}+\sigma^{3}-a \omega^{2}+\sigma \omega^{2}\right) \sin \omega=0$ & $\left.+\omega\left(2 a \sigma+\sigma^{2}+\omega^{2}\right) \sin \omega\right]$ \\
$s(s+a)+k(s+F) e^{-s}$ & $\omega\left(a F+2 F \sigma+\sigma^{2}+\omega^{2}\right) \cos \omega+$ & $\frac{-e^{\sigma}}{(F+\sigma)^{2}+\omega^{2}}[(\sigma(a+\sigma)(F+\sigma)$ \\
& $\left(\sigma(a+\sigma)(F+\sigma)+(a-F+\sigma) \omega^{2}\right) \sin \omega=0$ & $\left.+(a-F+\sigma) \omega^{2}\right) \cos \omega$ \\
& & $\left.-\omega\left(a F+2 F \sigma+\sigma^{2}+\omega^{2}\right) \sin \omega\right]$ \\
\hline
\end{tabular}

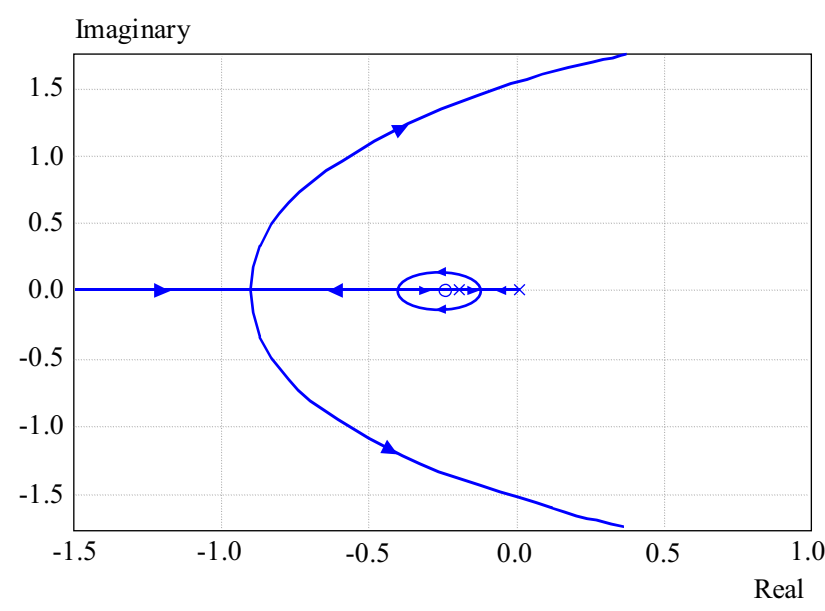

Fig. 3. The primary branches of a root locus diagram for a system with time delay, drawn for $k>0$, showing a more intricate topology than the Lambert $W$ function.

along the imaginary axis until it meets one of the infinite number of branches that start at $\sigma=-\infty$ and end at $\sigma=+\infty$. When these two branches meet they form the leftmost breakpoint. Figure 3 shows a more intricate topology than the Lambert $W$ function - this reflects the richer dynamics of the system modelled here.

\section{THE EQUIVALENCE BETWEEN \\ THE LAMBERT W FUNCTION AND THE ROOT LOCUS EQUATION}

\subsection{Solution to a transcendental equation in terms of the Lambert $W$ function}

The Lambert $W$ Function $W(z)$ may be used to solve a restricted type of transcendental equation [22-24]. This approach may be used when the equation is simple enough to be arranged in the form

$$
W(z) e^{W(z)}=z
$$

where $z$ is a complex number. More typically $[23,24]$ equation (13) takes the form

$$
W(z) e^{W(z)}=k
$$

where $k$ is a real number. Consider an example from [24]

$$
(s+\alpha)^{2}-k e^{-s L}=0 .
$$

The Lambert $W$ function approach may be used to solve (15) for $s$. First, by taking positive square roots (15) can be rearranged as

$$
\begin{aligned}
(s+\alpha)^{2} e^{s L}=k \Longrightarrow & (s+\alpha) e^{s L / 2}=\sqrt{k} \Longrightarrow \\
& \frac{L}{2}(s+\alpha) e^{\frac{L}{2}(s+\alpha)}=\frac{L}{2} \sqrt{k} e^{\frac{\alpha L}{2}} .
\end{aligned}
$$

We now have rearranged (15) as

$$
W(s) e^{W(s)}=\frac{L}{2} \sqrt{k} e^{\frac{\alpha L}{2}} .
$$

The term on the right hand side of (17) is a real number $K \in \Re^{1}$ so we can write

$$
W(s) e^{W(s)}=K .
$$

Finally

$$
\begin{aligned}
W(s)=\frac{L}{2}(s+\alpha) \Longrightarrow s & =\frac{2}{L} W(s)-\alpha \Longrightarrow \\
s & =\frac{2}{L} W\left(\frac{L}{2} \sqrt{k} e^{\frac{\alpha L}{2}}-\alpha\right)
\end{aligned}
$$

(19) is a closed-form expression for the roots of (15).

\subsection{Plotting the solutions to a transcendental equation using root locus}

It is a simple matter to rearrange (14) as

$$
W(z)-K e^{-W(z)}=0
$$

(20) is just (10), ie $s+k e^{-s}=0$, with the change of variables $s \rightarrow W(z)$ or $(\sigma+j \omega) \rightarrow(x+j y)$ - the negative sign on $K$ is irrelevant here. So the root locus of (10) in the $(\sigma, \omega)$-plane is the same as the root locus of $(20)$ in the $(x, y)-$ plane. Or to put it another way, the paths traced out in the $(\sigma, \omega)$-plane by the solutions to (10) are the same as the paths traced out in the $(x, y)$-plane by the solutions to (20). So the root locus diagram of $(20)$ in the $(x, y)$ - plane is a diagram of $W(z)[19]$. 


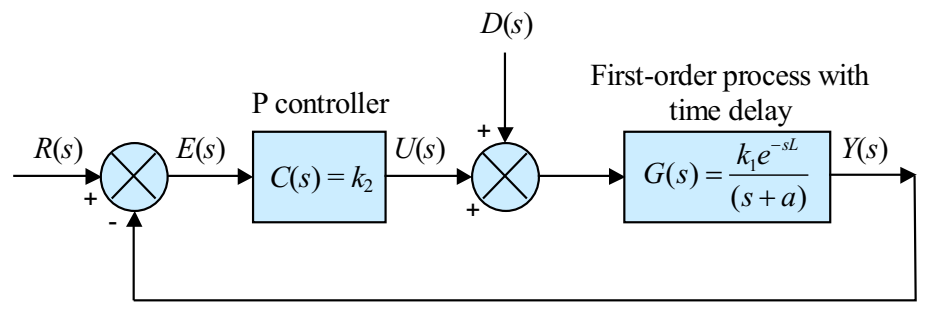

Fig. 4. Proportional controller and a first-order time-delay process with time delay $L>0$

Another way to see this is to substitute $W(z)=x+j y$ into (18) to get

$$
\begin{array}{r}
\left.W(z) e^{W(z)}=e^{x}\{x \cos y-y \sin y)+j(y \cos y+x \sin y)\right\} \\
=K \in \Re^{1} .
\end{array}
$$

Comparing the root locus equation (11) and the gain equation (12) with (21) we see that the imaginary part of (21) is the root locus equation (11) and the real part of (21) is the gain equation (12).

A comparison of the root locus of (10) given in Fig. 2 and the plot of the Lambert $W$ function given in, for example, [21] and [1] further illustrates this equivalence.

Table 1 gives illustrative examples of characteristic polynomials along with the corresponding root locus and gain equations.

\subsection{Discussion}

The root locus equation has been shown here to contain the Lambert $W$ function as a special case. This is not surprising as the root locus is a drawing of the roots of an equation and the Lambert $W$ function is a solution to the same equation. Unlike the Lambert $W$ function approach, when using the root locus approach to analyse solutions to transcendental equations it is not necessary for the equation to be simple enough to be transformed into (13) - the properties of the solutions to any equation of the form $p(s)=N(s)+k e^{-s L} M(s)$ can be studied with the root locus method. An illustrative example of such a study, amenable to analysis by both Lambert $W$ function and root locus, is given in the following section.

\section{ILLUSTRATIVE EXAMPLE OF ANALYTIC ROOT LOCUS AND LAMBERT W FUNCTION ANALYSIS}

\subsection{Analysis of a Proportional controller with a FOLPD process}

This illustrative example is studied here as it results in a characteristic equation that is simple enough to be analysed using the Lambert $W$ function approach. In fact a Proportional controller is rarely used with a FOLPD process as it will give a steady state error for a setpoint change. It can still be valuable however where disturbances are insignificant or are not sustained. A more realistic problem - designing a PI controller for an FOLPD process - cannot be analysed using the Lambert $W$ function approach but may be studied using root locus [20].

The transfer function for a first-order lag plus timedelay (FOLPD) process is given by

$$
G(s)=\frac{k_{1} e^{-s L}}{s+a}
$$

where $k_{1}$ is the steady-state process gain, $L>0$ is the time delay of the process. If $a \neq 0$ then $1 / a$ is the time constant of the process. " $a$ " can be positive (stable process), zero (an integrator), or negative (an unstable process).

The simple Proportional controller ( $\mathrm{P}$ controller) used here has transfer function $C(s)=k_{2}$, where $k_{2}$ is the gain of the controller. Figure 4 shows the $\mathrm{P}$ controller connected with a FOLPD process in a negative feedback configuration. Letting $k=k_{1} k_{2}$, the characteristic equation of this system is

$$
p(s)=(s+a)+k e^{-s L} .
$$

Equation (23) can be solved using the Lambert $W$ function approach:

$$
\begin{aligned}
& s+a=-k e^{-s L} \Longrightarrow(s+a) e^{s L}=-k \Longrightarrow \\
& \quad(s+a) L e^{(s+a) L}=-k L e^{a L} \text { or } W(s) e^{W(s)}=-k L e^{a L} .
\end{aligned}
$$

From $(24)$ we have $W(s)=(s+a) L \Longrightarrow s=(W(s) / L)-$ $a$. Now we can write the following closed-form expression for the roots of the characteristic equation (23)

$$
s=\left(W\left(-k L e^{a L} / L\right)-a .\right.
$$

Since $s=\sigma+j \omega$ and $W(s)=x+j y$ we can equate the real and imaginary parts of $W(s)=(s+a) L$ to get the following relationship between the $(\sigma, \omega)$-plane and the $(x, y)$-plane

$$
(\sigma, \omega)=\left(\frac{x}{L}-a, \frac{y}{L}\right)
$$

Using (26) we can transfer between equations (23) and (24). Also, (24) may be written as

$$
e^{x}\{(x \cos y-y \sin y)+j(y \cos y+x \sin y)\}=-L k e^{a L} .
$$




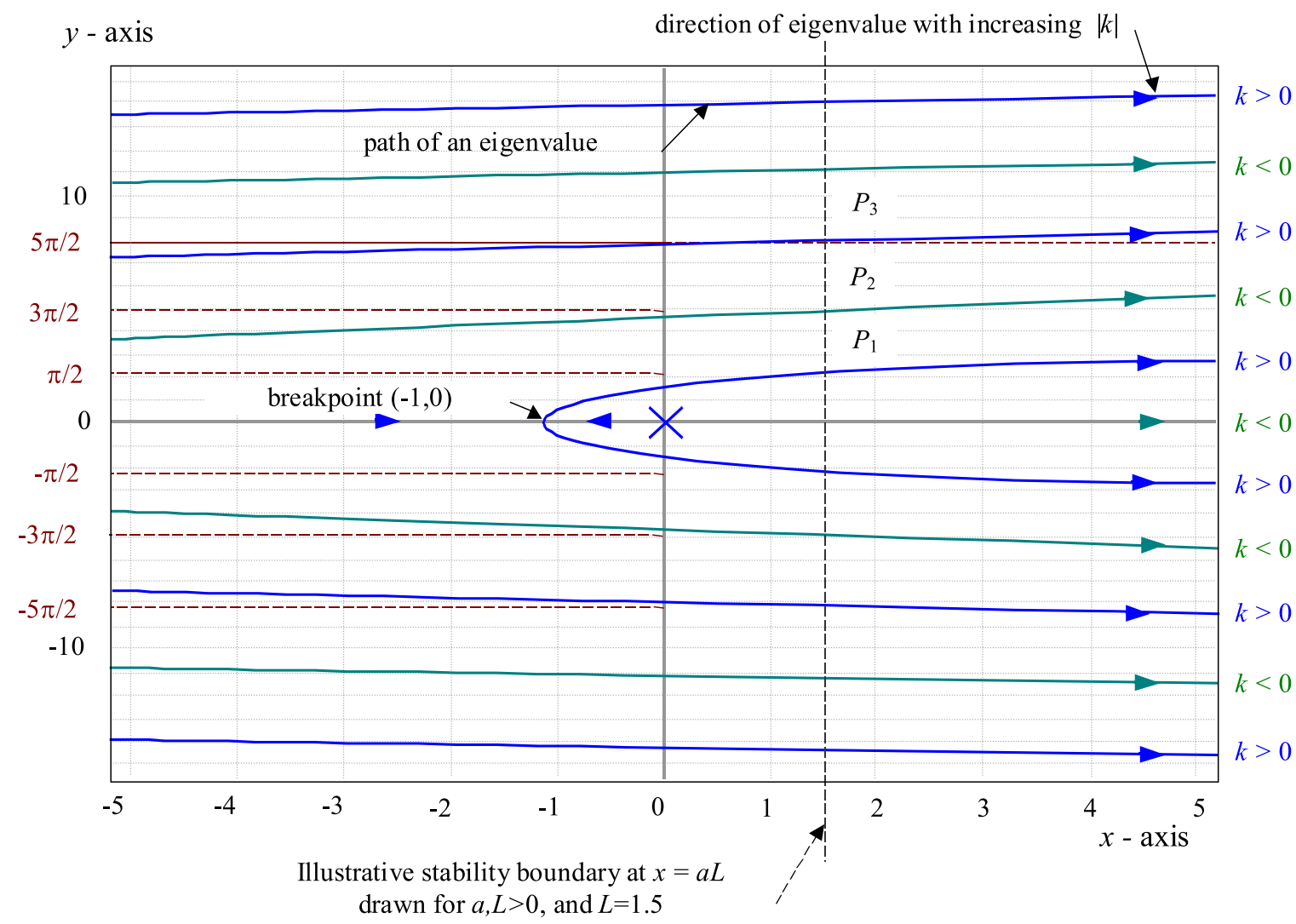

Fig. 5. Root locus diagram for (28) drawn in the $(x, y)$-plane. Positive parameter root locus $(k>0)$ and negative parameter root locus $(k<0)$ branches are given. The stability boundary at $x=a L$ corresponds to the $\omega$-axis in the $(\sigma, \omega)$-plane. Asymptotes are not shown but they consist of horizontal lines drawn between the root locus branches parallel to the $x$-axis and intersecting the $y$-axis at $y= \pm n \pi$.

Using $K=k L e^{a L}$ equation (24) an be rearranged as

$$
W(s)+K e^{-W(s)}=0 .
$$

Using either $-L k e^{a L} \in \Re^{1}$ and $(27)$ or the root locus equation (8) and gain equation (9) we can derive the root locus equation for (28)

$$
y \cos y+x \sin y=0
$$

and the gain equation for (28)

$$
e^{x}(x \cos y-y \sin y)=-L k e^{a L} .
$$

The root locus diagram for (28) is given by plotting (29) and is shown in Fig. 5.

Some properties of the root locus diagram of (23), given in Fig. 5, and hence some properties of the system in Fig. 4 will now be derived.

(a) Setting $x=0$ in (29) gives the point where the root locus intersects the $y$-axis

$y \cos y=0 \Longrightarrow y=0$ or $y= \pm \frac{(2 n+1) \pi}{2}, n=0,1,2, \ldots$

(b) As $|x| \rightarrow \infty$, (29) implies that the root locus branches approach $y= \pm n \pi$. So the asymptotes intersect the $y$-axis at $y \pm n \pi, n=0,1,2, \ldots$. (c) The stability boundary in the $(\sigma, \omega)$-plane is given by $\sigma=0$. In the $(x, y)$-plane the stability boundary is given by $\frac{x}{L}-a=0 \Longrightarrow x=a L$. So if $a L<-1$ the system cannot be stabilised as the breakpoint lies to the right of the stability boundary. This result was derived with more difficulty in [25] using the Nyquist criterion.

(d) The point on the root locus in Fig. 5 where two branches leave the $x$-axis is called a breakpoint. We can find the breakpoint by setting the derivative of the root locus equation to zero [26]

$$
\Longrightarrow \cos y-y \sin y+x \cos y=0 \text {. }
$$

(31) gives $(x, y)=(-1,0)$ as the breakpoint. Using $(26)$ we find the breakpoint in the $(\sigma, \omega)$-plane $((-1-a L) / L, 0)$.

(e) Root locus branches nearest to the real axis are called the primary branches, as these are the ones that cross the imaginary axis first with subsequent crossings taking place at larger values of $k$. These branches are critical when considering stability $[14,15]$.

A relationship between $\left|k_{z=j \omega}\right|$, the gains at which the branches cross the stability boundary, and the values of $y$ at the crossing points, can be established by rearranging 


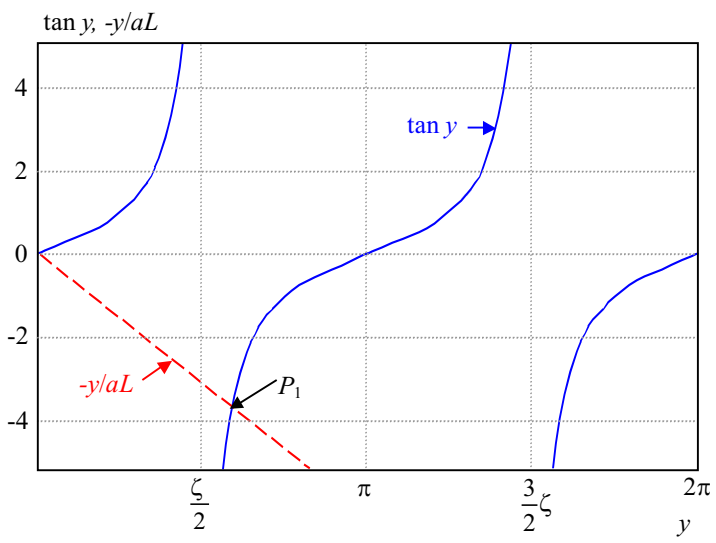

Fig. 6. Plots for $\tan y$ and the straight line $-y / a L$

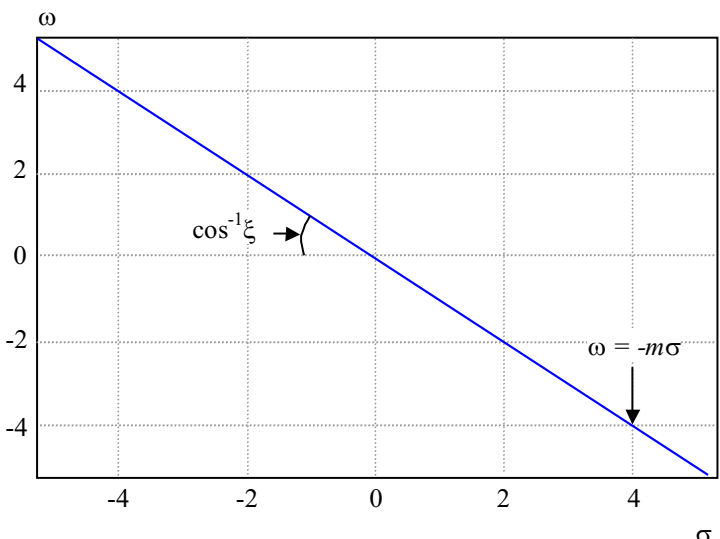

Fig. 7. The line $\omega=-m \sigma$ in the $(\sigma, \omega)$-plane

(29) and (30) evaluated at $x=a L$ as follows

$$
\begin{aligned}
& \left(\begin{array}{cc}
a L & -y \\
y & a L
\end{array}\right)\left(\begin{array}{l}
\cos y \\
\sin y
\end{array}\right)=\left(\begin{array}{c}
L k \\
0
\end{array}\right) \Longrightarrow \\
& \quad\left(\begin{array}{l}
\cos y \\
\sin y
\end{array}\right)=\frac{1}{a^{2} L^{2}+y^{2}}\left(\begin{array}{cc}
a L & y \\
-y & a L
\end{array}\right)\left(\begin{array}{c}
-L k \\
0
\end{array}\right) .
\end{aligned}
$$

Expanding (32) and invoking $\cos ^{2} y+\sin ^{2} y=1$ we find

$$
k=\frac{ \pm \sqrt{a^{2} L^{2}+y^{2}}}{L} .
$$

So for each crossing point we can see that $|k|$ increases monotonically with $|y|$. However, there is still an ambiguity - for example, if $a=1, L=3$ and $y= \pm 4$, (33) gives $k= \pm 5$. So crossings of the line $x=a L$ occur in pairs - each value of $k$ can have two values for $y$. This ambiguity may be removed by substituting (29) into (30) to obtain

$$
\begin{aligned}
&-\frac{y \cos y}{\sin y} \cos y-y \sin y=-L k e^{(a L-x)} \Longrightarrow \\
& \frac{y}{\sin y}=L k e^{(a L-x)} .
\end{aligned}
$$

Since $\operatorname{sign}[y / \sin y]$ alternates along the $y$-axis, we know from (33) and (34) that higher values of $|k|$ imply higher values of $|y|$; if a pair of crossings of the $x=a L$ line occur for some $k_{i}>0$ the next pair of crossings will take place for some $k_{i+1}<0$ where $\left|k_{i+1}\right|>\left|k_{i}\right|$ and the following pair of crossings occur for some $k_{i+2}>0$ where $\left|k_{i+2}\right|>\left|k_{i+1}\right|>\left|k_{i}\right|$, etc. These root locus branches comprise the positive and negative parameter root locus.

(f) The stability limit is reached at $x=a L>0$ and at this point (29) becomes

$$
y \cos y+a L \sin y=0 .
$$

(35) can be rearranged as follows

$$
\tan y=-y /(a L) .
$$

Figure 6 shows that with $a L>0$ the appropriate root for $y$ is in the range $\frac{\pi}{2}<y<\pi$. At the point $P_{1}$ in Fig. 6, the following equation holds

$$
\tan y=-y /(a L) \Longrightarrow y=\tan ^{-1}(-y / a L)+\pi .
$$

Consider the iterative algorithm

$$
y_{k+1}=\tan ^{-1}\left(-y_{k} / a L\right)+\pi .
$$

With $a L=0.5$ and an initial point of $y_{1}=2$ the iterative algorithm in $(38)$ converges to $y_{4}=1.836 \ldots$ At the point $P_{1}(x, y)=(0.5,1.836 \ldots)$ and $(35)$ gives $k=$ -3.806 - as can be confirmed using (33). If $k>3.806$ the system is unstable.

\subsection{Two designs for a Proportional controller with a FOLPD process}

\subsubsection{Controller design using optimum stability}

When the system parameter is such that the rightmost eigenvalue is as far to the left as possible in the $(\sigma, \omega)$ plane we can say that the system is in a state of optimum stability in the root locus sense $[19,20,27]$. In the Lambert $W$ function plane the optimum stability point of the system in Fig. 5 is the breakpoint in the root locus: $(x, y)=(-1,0)$. At this optimum stability point, and with $a=1$ and $L=0.5$, the gain equation (30) gives

$$
k=\frac{-(x \cos y-y \sin y) e^{(x-a L)}}{L}=\frac{e^{-(a L+1)}}{L}=0.4462 \ldots
$$

Now we have to show that if $k=k_{\text {opt }}=0.4462 \ldots$ $i e$ if we are operating at the point of optimum stability $(x, y)=(-1,0)$, then the values of $k_{y}$ at $(-1, y), y>0$, are all greater than $k_{\mathrm{opt}}$.

At the point $(-1, y)$ the root locus equation (29) gives

$$
y \cos y-\sin y=0
$$

and the gain equation (30) gives

$$
-\cos y-y \sin y=-L k e^{(a L+1)} .
$$




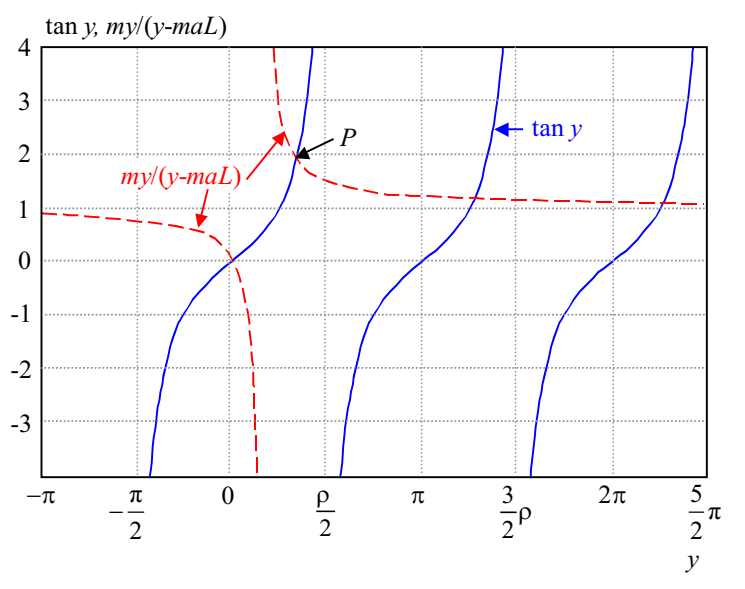

Fig. 8. $\tan y$ and $\frac{m y}{y-m a L}$ drawn for $m a L<\frac{\pi}{2}, m=1 \Longrightarrow \zeta=$ $1 / \sqrt{2}, L=0.5$

(40) and (41) may be written as

$$
\begin{gathered}
\left(\begin{array}{rr}
-1 & -y \\
y & -1
\end{array}\right)\left(\begin{array}{l}
\cos y \\
\sin y
\end{array}\right)=\left(\begin{array}{c}
-L k e^{(a L+1)} \\
0
\end{array}\right) \Longrightarrow \\
\left(\begin{array}{l}
\cos y \\
\sin y
\end{array}\right)=\frac{1}{1+y^{2}}\left(\begin{array}{rr}
-1 & y \\
-y & -1
\end{array}\right)\left(\begin{array}{c}
-L k e^{(a L+1)} \\
0
\end{array}\right)
\end{gathered}
$$

Expanding (42) and invoking $\cos ^{2} y+\sin ^{2} y=1$ and taking the positive root locus branches gives

$$
k=\frac{\sqrt{1+y^{2}}}{L} e^{-(a L+1)}=\sqrt{1+y^{2}} k_{\mathrm{opt}} .
$$

So the point $(-1,0$ is indeed the point of optimum stability. In summary, when $k=0.4462$ the rightmost eigenvalue is as far to the left as possible, ie at $(-1,0)$, and the system is operating at a point of optimum stability [19]. This design gives Gain Margin (GM) of $18.6 \mathrm{~dB}$.

\subsubsection{Controller design using the dominant eigenvalue} method

The dominant eigenvalue approach to controller design is to choose that value of $k$ such that the eigenvalues closest to the origin in the principal strip have a specified damping ratio $\zeta, 0<\zeta<1$.

From Fig. 7 we have $\zeta=1 / \sqrt{1+m^{2}}$ so $m=1$ gives damping ratio $\zeta=1 / \sqrt{2}$. Also, the line $\omega=-m \sigma$ in the $(\sigma, \omega)$-plane maps to the line $\frac{Y}{L}=-m\left(\frac{x}{L}-a\right)$ in the $(x, y)$-plane. The equation for this line may be rearranged as

$$
-\frac{y}{x}=\frac{-y}{-(y / m)+a L} .
$$

The root locus equation is

$$
y \cos y+x \sin y=0 .
$$

(44) may be rearranged as

$$
-\frac{y}{x}=\tan y .
$$

(43) and (45) may be solved for $y$ by first noting

$$
\tan y=\frac{m y}{y-m a L} .
$$

Figure 8 shows the curves for $\tan y$ and $\frac{m y}{y-m a L}$ on the same axes.

The iterative algorithm

$$
y_{k+1}=\tan ^{-1} \frac{m y_{k}}{y_{k}-m a L}
$$

with values $m=1, a L=0.5$, and initial value $y_{1}=1$, stabilises to nine decimal places after twenty iterations giving $y=1.0784 \ldots$ at the point $P$. Now we can use (44) to get $x=-0.5784 \ldots$ and the gain equation (39) to calculate $k=0.8324 \ldots$ ie the value of $k$ that ensures that the eigenvalues closest to the origin in the principal strip have damping ratio $\zeta=1 / \sqrt{2}$.

At the stability boundary $k=3.8068 \ldots$ so the gain margin for the dominant eigenvalue design is

$$
\mathrm{GM}=20 \log _{10} \frac{3.8068 \ldots}{0.8324 \ldots} \mathrm{dB} \cong 13.2 \mathrm{~dB} .
$$

\section{CONCLUSIONS}

The root locus equation has been shown to contain the Lambert $W$ function as a special case. Also, the root locus is seen to be applicable to a far wider range of systems than the Lambert $W$ function. Since the system eigenvalues are central to the root locus approach, it can offer deeper physical insight.

As an illustrative example, the analytic root locus and Lambert $W$ function approaches are used to analyze, in detail, a system comprising a Proportional controller with a time-delay process and several interesting and useful properties are derived. Controllers for this system are designed using two techniques — one based on optimum stability and the other on the dominant eigenvalue method. The design based on optimum stability gives greater Gain Margin.

\section{REFERENCES}

[1] CORLESS, R. M.-GONNET,-HARE, G. H.-JEFFREY, D. J.-KNUTH, D. E. : On the Lambert $W$ Function,, Advances in Computational Mathematics 5 (1996), 329-359.

[2] ANDERSEN, K.: The Geometry of Art - The History of the Mathematical Theory of Perspective from Alberti to Monge, Springer, 2007.

[3] SENGUPTA, P.: The Lambert $W$ Function and Solutions to Kepler's Equation, Celestial Mechanics and Dynamical Astronomy 99 (2007), 13-22.

[4] FURSE, C. : Applications of the Lambert $W$ Function in Electromagnetics, IEEE Antennas and Propagation Magazine 44 (2002), 139-142. 
[5] WARBURTON, R. D. H.: An Analytical Investigation of the Bullwhip Effect, Production and Operations Management 13 (2004), 150-160.

[6] VALLURI, S. R.-GIL, M.-JEFFREY, D. J.-BASU, S. : The Lambert $W$ Function and Quantum statistics, Journal of Mathematical Physics 50 (2009), 102-111.

[7] WILLIAMS, B. W. : Exact Solutions to a Schrodinger Equation Based on the Lambert Function, Physics Letters A 334 (2005), 117-222.

[8] CRANMER, S. R.: New Views of the Solar Wind with the Lambert W Function, American Journal of Physics 72 (2004), 1397-1403.

[9] Maplesoft, Maple, 615 Kumpf Drive, Waterloo, Ontario, Canada.

[10] MathWorks, MatLab, The Math Works, Inc., 3 Apple Hill Drive, Natick, MA 01760-2098, USA.

[11] WolframReseach, Mathematica, Wolfram Research Inc., 100 Trade Canter Drive, Champaign, IL 61820, USA.

[12] POWER, H. M.-SIMPSON, R. J. : Introduction to Dynamics and Control, McGraw-Hill, 1978.

[13] D'AZZO, J. J.-HOUPIS, C. H. : Linear Control System Analysis and Design Conventional and Modern, Mc Graw Hill, New York, 1988

[14] OGATA, K. : Modern Control Engineering, Prentice Hall, 2002.

[15] PALM, W. J. III : Control System Engineering, John Wiley \& Sons, 1986.

[16] BENDRIKOV, G. A.-TEODORCHIK, K. F.- : The Analytic Theory of Constructing Root Loci, Automation and Remote Control 20 (1959), 340-344.

[17] COGAN, B.: Use of the Analytic Method and Computer Algebra to Plot Root Loci, International Journal of Electrical Engineering Education 35 (1998), 350-356.

[18] KING-SMITH, E. A.: Stability Analysis of Linear Continuous Time-Delay Feedback Systems, International Journal of Control 13 (1971), 633-655.

[19] COGAN, B.: Optimum Stability in Control System Design, $\mathrm{PhD}$ thesis, Department of Electronic and Electrical Engineering, Trinity College, Dublin, 2006.

[20] COGAN, B.-de PAOR, A. M.-QUINN, A.: PI Control of First-Order Lag Plus Time-Delay Plants, Transactions of the Institute of Measurement and Control 31 (2009), 365-379.

[21] SHINOZAKI, H.-MORI, T. : Robust Stability Analysis of Linear Time-Delay Systems by Lambert $W$ Function: Some Extreme Point Results, Automatica 42 (2006), 1791-1799.

[22] WANG, Z. H.-HU, H. Y. : Calculation of the Rightmost Characteristic Root of Retarded Time-Delay Systems via Lambert $W$ Function, Journal of Sound and Vibration 318 (2008), 757-767.
[23] HWANG, C.-CHENG, Y.-C.: A Note on the Use of the Lambert $W$ Function in the Stability Analysis of Time-Delay Systems, Automatica 41 (2005), 1979-1985.

24] CHEN, Y.-MOORE, K. L. : Analytic Stability Bound for Delayed Second-Order Systems with Repeating Poles Using Lambert Function $W$, Automatica 38 (2002), 891-895.

25] de PAOR, A. M.-O'MALLEY, M. J.: Controllers of the Ziegler-Nichols Type for Unstable Process with Time Delay, International Journal of Control 49 (1989), 1273-1284.

26] STEFANI, R. S.-SHAHIAN, B.-SAVANT, C. J.-HOSTETTER, G. H.: Design of Feedback Control Systems, Oxford University Press, 2002.

27] de PAOR, A. M.: Concepts of Optimum Stability for Linear Feedback Systems, International Journal of Electrical Engineering Education 36 (1999), 46-64.

Received 27 March 2011

Brian COGAN obtained the degrees of BE (1979) and MEngSc (1986) from National University of Ireland, Dublin, and PhD (2006) from Trinity College Dublin for research in Control Engineering. He worked as a lecturer and as a consulting engineer for several years and is now a Projects Manager with the Commissioners of Irish Lights. He is a Member of The Institution of Engineering and Technology.

Annraoi M de PAOR obtained the degrees of BE (1961), PhD (1967) and DSc (1974) from the National University of Ireland, Dublin, all in Electrical Engineering, and the MS from the University of California at Berkeley (1963). He lectured at the University of Salford, UK, from 1963 to 1967, and the National University of Ireland, Dublin, from 1967 to 1969. In 1969, he was appointed Professor of Control Engineering at the University of Salford and on 1 January 1978 took up the position as Professor of Electrical Engineering at the National University of Ireland, Dublin. Since September 2005 he is Professor Emeritus. He has published in Control Theory, Biomedical Engineering, Renewable Energy Systems, Classical Electrical Engineering, Geomagnetism and Engineering Education. He is a Fellow of The Institution of Engineering and Technology, The Institution of Engineers of Ireland and The Institute of Mathematics and its Applications, and is a Member of the Royal Irish Academy, and the Institute of Measurement and Control. 